\title{
Boson-boson scattering and Higgs production at the LHC from a six fermion point of view: four jets $+1 \nu$ processes at $\mathcal{O}\left(\alpha_{\text {em }}^{6}\right)$.
}

\author{
E. Accomando ${ }^{1,2}$, A. Ballestrero ${ }^{1,2}$, S. Bolognesi ${ }^{1,3}$, E. Maina ${ }^{1,2}$, C. Mariotti $^{1}$ \\ ${ }^{1}$ INFN, Sezione di Torino, Italy \\ ${ }^{2}$ Dipartimento di Fisica Teorica, Università di Torino, Italy \\ ${ }^{3}$ Dipartimento di Fisica Sperimentale, Università di Torino, Italy
}

June 17, 2021

\begin{abstract}
Boson-boson scattering and Higgs production in boson-boson fusion hold the key to electroweak symmetry breaking. In order to analyze these essential features of the Standard Model we have performed a partonic level study of all processes $q_{1} q_{2} \rightarrow q_{3} q_{4} q_{5} q_{6} l \nu$ at the LHC using the exact matrix elements at $\mathcal{O}\left(\alpha_{e m}^{6}\right)$ provided by PHASE, a new MC generator. These processes include also three boson production and the purely electroweak contribution to t $\bar{t}$ production as well as all irreducible backgrounds. Kinematical cuts have been studied in order to enhance the VV scattering signal over background. PHASE has been compared with different Monte Carlo's showing that a complete calculation is necessary for a correct description of the process.
\end{abstract}

DFTT $37 / 2005$

E.A. is supported by the Italian Ministero dell'Istruzione, dell'Università e della Ricerca (MIUR) under contract Decreto MIUR 26-01-2001 N.13 "Incentivazione alla mobilità di studiosi stranieri ed italiani residenti all'estero".

Work supported by MIUR under contract 2004021808_009. 


\section{Introduction}

The nature of Electro-Weak Symmetry Breaking (EWSB) will be investigated at the $\mathrm{LHC}^{1}$. The Standard Model (SM) provides the simplest and most economical explanation of the phenomenon and all its predictions have been verified with unprecedented accuracy. The only missing ingredient is the Higgs boson which is essential to the renormalizability of the theory. The fit of precision EW data to the SM currently gives an upper limit on the Higgs mass of about $200 \mathrm{GeV}$, depending on the preferred value of the top mass [5]. A heavier Higgs mass can however be accomodated by a number of models which in general would be distinguishable from the SM by improved precision data or by other observations at future colliders [6]

In the SM the Higgs is also crucial to ensure that perturbative unitarity bounds are not violated in high energy reactions. Scattering processes between longitudinally polarized vector bosons are particularly sensitive in this regard and without a Higgs they violate unitarity at about one $\mathrm{TeV}$.

It should be noted that the Goldstone theorem and the Higgs mechanism do not require the existence of elementary scalars. It is conceiveable and widely discussed in the literature that bound states are responsible for EWSB.

Since unitarity is essentially a statement of conservation of total probability it cannot be violated in Nature. Violation of perturbative unitarity implies that the SM becomes a strongly interacting theory at high energy. The nature of the interaction between longitudinally polarized vector bosons and the Higgs mass, or possibly the absence of the Higgs particle, are strongly related: if a relatively light Higgs exists than the $\mathrm{V}_{\mathrm{L}}$ 's are weakly coupled, while they are strongly interacting if the Higgs mass is large or the Higgs is nonexistent [7]. In the latter case, by analogy with low energy QCD, which can be expressed by exactly the same formalism which describes the Higgs sector in the SM, or adopting one of the many schemes for turning perturbative scattering amplitudes into amplitudes which satisfy by construction the unitarity constraints, one is led to expect the presence of resonances in $\mathrm{W}_{\mathrm{L}} \mathrm{W}_{\mathrm{L}}$ scattering. Unfortunately the mass, spin and even number of these resonances are not uniquely determined [8]. If, on the other hand, a Higgs particle is discovered it will nonetheless be necessary to verify that indeed longitudinally polarized vector bosons are weakly coupled at high energy by studying boson boson scattering in full detail. If the Higgs mass is not far from the present experimental lower limits it will take several years of data taking before enough statistics could be accumulated for a reliable discovery. In the meantime high energy vector boson scattering could provide an alternative method for investigating the existence of a light Higgs scalar, providing a strong incentive to probe harder the low mass range if no sign of strong scattering is detected.

At the LHC no beam of on shell EW bosons will be available. Incoming quarks will emit spacelike virtual bosons which will then scatter among themselves and finally decay. These processes have been scrutinized since a long time [9, 10]. All previous studies of boson boson scattering at high energy hadron colliders have resorted to some approximation, either the Equivalent Vector Boson Approximation (EVBA) [11], or a production times decay approach, supplementing a calculation of

$$
q_{1} q_{2} \rightarrow q_{3} q_{4} V_{1} V_{2}
$$

\footnotetext{
${ }^{1}$ Detailed reviews and extensive bibliographies can be found in Refs.[1, 2, 3, 4]
} 
processes with the on shell decay of the two vector bosons. This is not surprising since the number of reactions required by a full six fermion calculation is very large and often approximate results are adequate for a first discussion. There are however issues that cannot be tackled without a full six fermion calculation like exact spin correlations between the decays of different heavy particles, the effect of the non resonant background, the relevance of off mass shellness of boson decays, the question of interferences between different subamplitudes. Without a complete calculation it will be impossible to determine the accuracy of approximate results.

Recently a full fledged six fermion Monte Carlo has become available [12], based on the methods of Refs. [13, 14], which in its present version describes at $\mathcal{O}\left(\alpha_{e m}^{6}\right)$, using exact matrix elements, all processes of the form

$$
P P \rightarrow q_{1} q_{2} \rightarrow q_{3} q_{4} q_{5} q_{6} l \nu
$$

(where $q_{i}$ stands for a generic (anti)quark) which can take place at the LHC. This provides a complete description of VV scattering and its EW irreducible background. Eq.(2) includes about one thousand different reactions. A complete classification of all processes can be found in [12]. Preliminary results have been presented in Refs. [15].

Since in addition to $\mathrm{V}_{\mathrm{L}} \mathrm{V}_{\mathrm{L}}$ scattering many other subprocesses are in general present in the full set of diagrams, it is not a trivial task to separate boson boson scattering from the EW irreducible background. In practice one has to deal also with other types of background to which QCD interactions contribute, but which however do not include any boson boson scattering term. We will refer to these processes as QCD background even though in general they will be a mixture of QCD and EW interactions. tt production and decay is particularly relevant and dangerous. In this paper we are neglecting QCD backgrounds, whose calculation is in progress, and concentrate on $\mathcal{O}\left(\alpha_{e m}^{6}\right)$ processes. It is clear that obtaining a good signal over EW irreducible background ratio is a prerequisite to any attempt at dealing with the QCD one.

In the absence of firm predictions in the strong scattering regime, trying to gauge the possibilities of discovering signals of new physics at the LHC requires the somewhat arbitrary definition of a model of $\mathrm{V}_{\mathrm{L}} \mathrm{V}_{\mathrm{L}}$ scattering beyond the boundaries of the SM. Some of these models predict the formation of spectacular resonances which will be easily detected. For some other set of parameters in the models only rather small effects are expected [8].

The simplest approach is to consider the SM in the presence of a very heavy Higgs. While this entails the violation of perturbative unitarity, the linear rise of the cross section with $\hat{s}$, the invariant mass squared in the hard scattering, will be swamped by the decrease of the parton luminosities at large momentum fractions and, as a consequence, will be particularly challenging to detect. At the LHC, for $M_{H}>10 \mathrm{TeV}$, all Born diagrams with Higgs propagators become completely negligible in the Unitary gauge, and the expectations for all processes in Eq.(2) reduce to those in the $M_{H} \rightarrow \infty$ limit. In this paper we will compare this minimalistic definition of physics beyond the Standard Model with the predictions of the SM for Higgs masses within the reach of the LHC. 

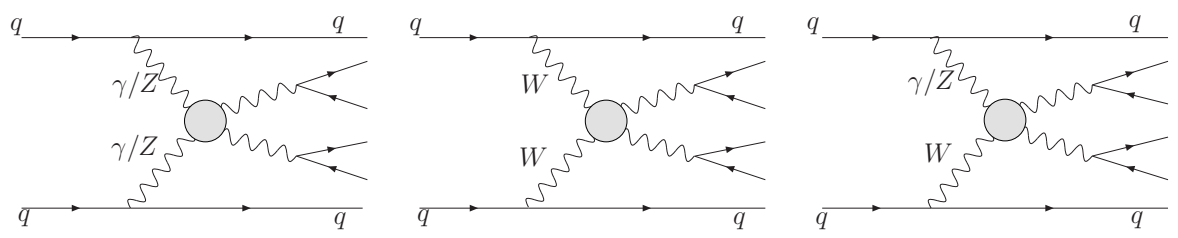

Figure 1: Vector boson fusion processes
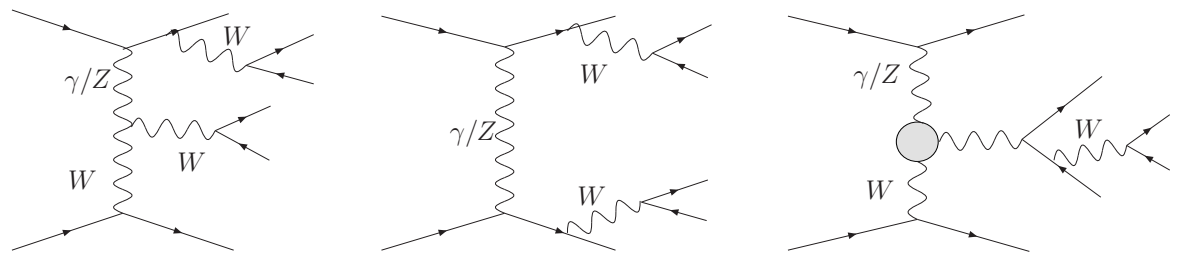

Figure 2: Non fusion and non doubly resonant two vector boson production.
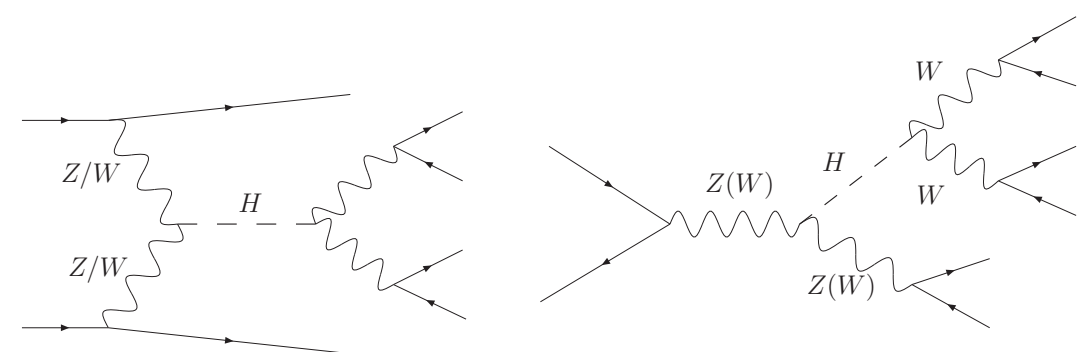

Figure 3: Higgs boson production via vector boson fusion and Higgstrahlung.
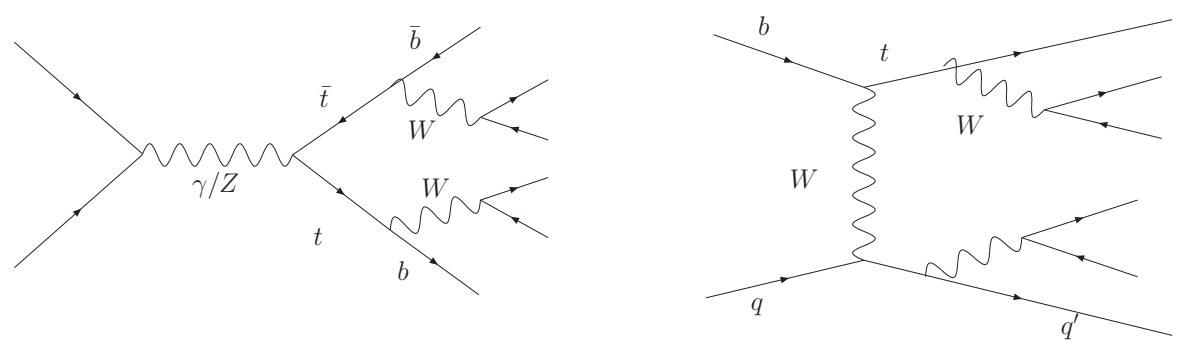

Figure 4: Electroweak t $\bar{t}$ and single top production. 


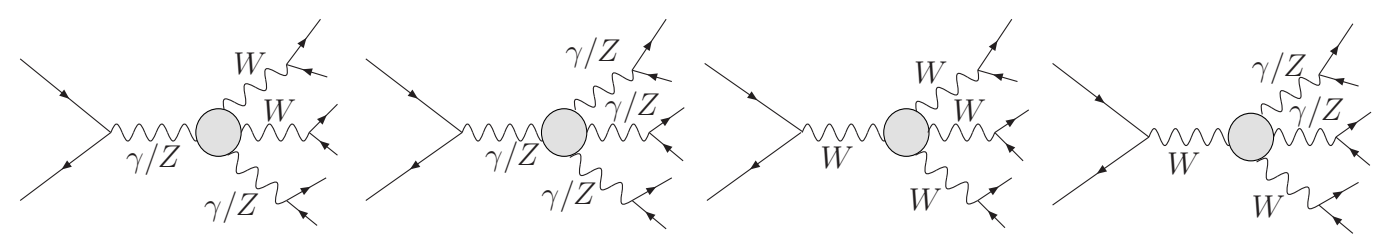

Figure 5: Three vector boson production.

\begin{tabular}{|c|}
\hline $\mathrm{E}$ (lepton) $>20 \mathrm{GeV}$ \\
\hline $\mathrm{p}_{\mathrm{T}}($ lepton $)>10 \mathrm{GeV}$ \\
\hline $\mid \eta($ lepton $) \mid<3$ \\
\hline $\mathrm{E}($ quarks $)>20 \mathrm{GeV}$ \\
\hline $\mathrm{p}_{\mathrm{T}}($ quarks $)>10 \mathrm{GeV}$ \\
\hline $\mid \eta($ quark $) \mid<6.5$ \\
\hline $\mathrm{M}(\mathrm{qq})>20 \mathrm{GeV}$ \\
\hline
\end{tabular}

Table 1: Standard acceptance cuts applied in all results. Any pair of colored fermions must have mass larger than $20 \mathrm{GeV}$. Here lepton refers to $l^{ \pm}$only.

\section{The six fermion final state processes}

Boson-boson scattering and Higgs production in boson-boson fusion produce intermediate states with two bosons and two quarks as shown in Fig. 1. In this study we have only considered final states in which one $\mathrm{W}$ boson decays leptonically and the other (either $\mathrm{Z}$ or W) hadronically, which is regarded as the best channel for probing these processes at the LHC [3]. If both bosons decay hadronically the signal cannot be distinguished from the QCD non resonant background whose cross section is much larger. Final states where both vectors decay leptonically have a smaller rate and have been left for future studies.

Once vector bosons are decayed we have six fermion final states. Since the V's emitted by the initial state particles are spacelike, we are forced by gauge invariance to include all diagrams in which final state V's are emitted directly by the fermion lines as in the left part of Fig. 2. When the finite width of the EW boson is properly taken into account and the outgoing vector bosons are allowed to be off mass shell, it becomes necessary, again because of gauge invariance, to consider the full set of diagrams described by Eq.(2). As a consequence, in addition to the diagrams which are related to the process we would like to measure, VV fusion, shown in Fig. 1, there will be diagrams in which a pair of V's are produced without undergoing VV scattering, as presented in Fig. 2. Furthermore, diagrams related to Higgs production via Higgstrahlung will also be present as shown in

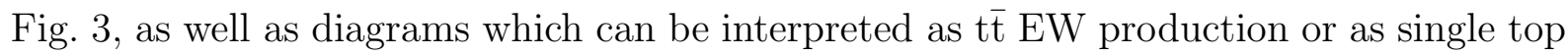
production as shown in Fig. 4. Finally diagrams describing three vector boson production which include Triple Gauge Coupling and Quartic Gauge Coupling will contribute as well, as shown in Fig. 5, since they produce the same kind of six fermion final states.

Depending on the flavour of the quarks in Eq.(2) the various subprocesses will contribute and interfere to a different degree. All processes will be experimentally indistin- 
guishable, apart from heavy quark tagging, and will have to be studied simultaneously.

A number of samples of events representative of all possible processes in Eq.(2) have been produced with PHASE. In order to comply with the acceptance of the CMS detector and with the CMS trigger requirements, the cuts in Tab. 1 have been applied. We have used the CTEQ5L [16] PDF set with scale

$$
Q^{2}=M_{W}^{2}+\frac{1}{6} \sum_{i=1}^{6} p_{T i}^{2}
$$

where $p_{T i}$ denotes the transverse momentum of the $i-$ th final state particle.

\subsection{Physical sub-processes}

As already mentioned many subprocesses (i.e. $\mathrm{WW} \rightarrow \mathrm{WW}, \mathrm{ZW} \rightarrow \mathrm{ZW}, \mathrm{ZZ} \rightarrow \mathrm{WW}, \mathrm{t} \overline{\mathrm{t}}$ ) will in general contribute to a specific six fermion reaction. It is impossible to separate and compute individually the cross section due to a single subprocess, since there are large interference effects between the different contributions.

We can however select all complete $2 \rightarrow 6$ processes which include a specific set of subdiagrams. For instance, ZW $\rightarrow \mathrm{ZW}$ with on shell bosons is described by 4 Feynman diagrams. These same diagrams, with all external vector bosons connected to a fermion line, constitute the ZW $\rightarrow$ ZW set of $2 \rightarrow 6$ diagrams. Several sets can contribute to a single process and therefore the same process can appear in different groups. The upper part of Fig. 6 shows the invariant mass distribution of the two most central quarks (when ordered in pseudorapidity $\eta$ ), the lepton and the neutrino for the reactions which contain the different subprocesses as well as the distribution for the complete set of processes. We assumed $\mathrm{M}(\mathrm{H})=500 \mathrm{GeV}$. For this figure, in addition to the standard cuts decribed in Tab. 1, the following cuts have been applied. The pseudorapidity of the two most central quarks should satisfy $\mid \eta$ (central-quark) $\mid<3$, the pseudorapidity of the two most forwardbackward quarks are constrained to $\mid \eta$ (forward-quark) $\mid>1$ and the transverse mass of the lepton neutrino pair should be smaller than $100 \mathrm{GeV}$. These three last requirements have been released for the studies presented in the following sections.

It should be clear that the total cross section in Fig. 6 is smaller than the sum of the cross sections for the various groups. Notice that the Higgs peak is present in the ZW $\rightarrow \mathrm{ZW}$ curve. This is due to processes that in addition to the ZW $\rightarrow \mathrm{ZW}$ set of diagrams include also diagrams describing Higgs production, e.g. $u \bar{u} \rightarrow u \bar{u} u \bar{d} \mu^{-} \bar{\nu}$.

The groups comprising single top and t $\bar{t}$ diagrams have a large cross section. An invariant mass analysis reveals that the processes in these two groups are indeed dominated by top production. The lower part of Fig. 6 shows the same distributions after subtraction of single top and t $\bar{t}$ and with the additional requirement that the two most central quarks have invariant mass between 60 and $100 \mathrm{GeV}$.

Top candidates are identified requiring a b-quark and two other quarks in the final state of the right flavour combination to be produced in a $\mathrm{W}$ decay, with a total invariant mass between 160 and $190 \mathrm{GeV}$. Analogously, events in which the muon, the neutrino and a b quark have an invariant mass between 160 and $190 \mathrm{GeV}$ are rejected. These vetoes reduce drastically the EW top background and produce a much sharper Higgs peak. 

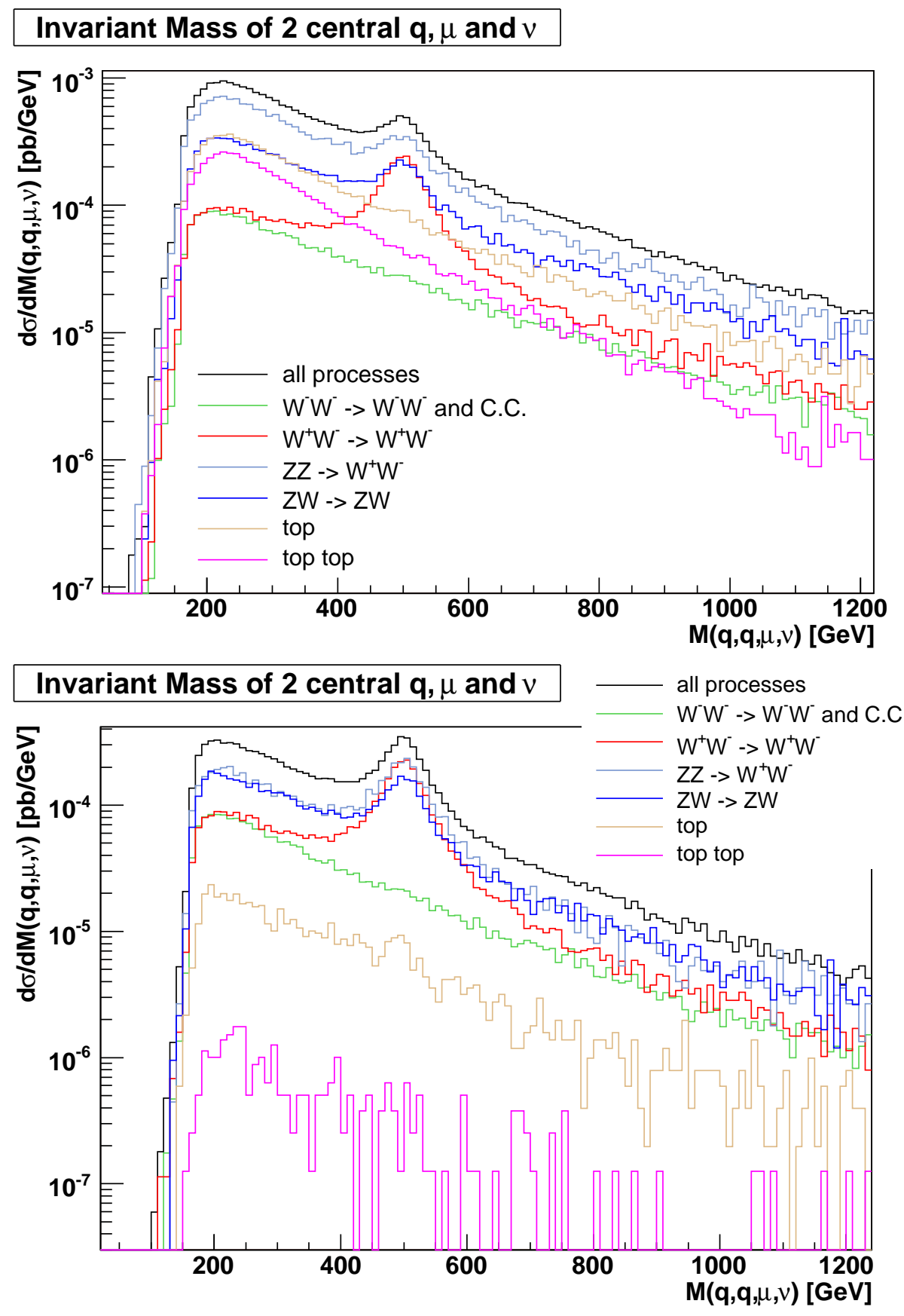

Figure 6: Invariant mass distribution of the lepton, neutrino and the two most central quarks, for different sets of processes. In the upper part with the set of cuts described in the text, in the lower part after vetoing top and $t \bar{t}$ production and requiring the vector bosons to be close to their mass shell. 


\section{Invariant Mass of bosons}

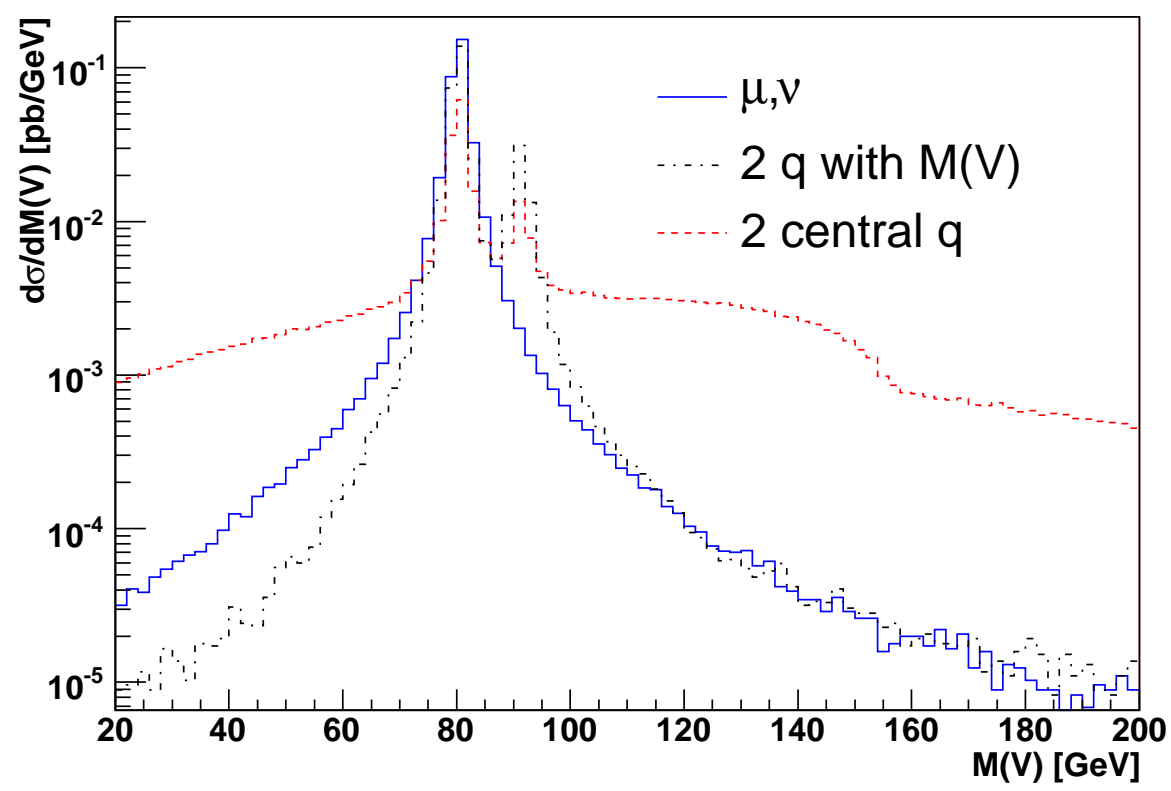

Figure 7: The invariant mass of the two most central quark in pseudorapidity (red/dashed) and for the quarks that gives the best nominal vector boson mass (black/dash-dotted) with PHASE. The invariant mass of the lepton and neutrino pair is shown in blue/continous for comparison.

\subsection{Identification of the hadronically decaying vector boson}

In the LHC environment it is not obvious how to identify the jets produced by the hadronic decay of an EW vector boson among the four which are present at generator level in the processes we are interested in. Fig. 7 shows the invariant mass of the candidate vector boson selected with two different procedures. In the first one final state quarks are ordered in pseudorapidity. The quarks with the largest and smallest $\eta$ are identified as tag quarks, while the remaining two, which will be referred to as "most central" quarks, are identified as the quarks from the $\mathrm{W}$ or $\mathrm{Z}$ decay $^{2}$. In the second procedure the two quarks among the four that have the mass closest to a vector boson mass are identified as the quarks from the decay while the remaining two are identified as tag quarks. As a comparison the invariant mass of the lepton and neutrino pair is also shown. It is clear from the figure that the second choice reproduces much better the lepton-neutrino mass distribution, giving higher efficiency and purity at generator level, and will be adopted in the rest of this paper.

\footnotetext{
${ }^{2}$ Notice that these two quarks are actually those with smaller $|\eta|$ only in the center of mass of the hard reaction and not in the laboratory frame. However the ordering in pseudorapidity is preserved by boosts along the beam line and the selection of the two jets with intermediate rapidity will be the same in the two reference frames
} 


\section{The VV-fusion signal}

In order to isolate the $\mathrm{VV}$ fusion process from all other six fermion final states and investigate EWSB, different kinematical cuts have been applied to the simulated events.

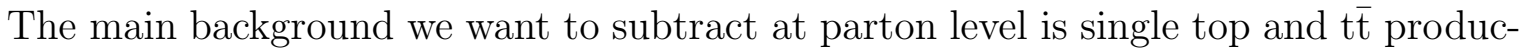
tion. These events have been identified as in Sub-Sect. 2.1 and rejected.

The invariant mass of the muon and the neutrino has to reconstruct the mass of a $\mathrm{W}$, and is required to be in the range $M_{W} \pm 10 \mathrm{GeV}$. In VV fusion an additional W or a $\mathrm{Z}$ decaying hadronically is expected to be present. Therefore events are required to contain two quarks with the correct flavours to be produced in $\mathrm{W}$ or $\mathrm{Z}$ decay, with an invariant mass of $\pm 10 \mathrm{GeV}$ around the central value of the appropriate EW bosons. If more than one combination of two quarks satisfies these requirements, the one closest to the corresponding central mass value is selected. This combination will in the following be assumed to originate from the decay of an EW vector boson.

In a second step, in order to reject events which can be identified with the production of three vector bosons, the flavour content and the invariant mass of the two remaining quarks is compared with a $\mathrm{W}$ and a $\mathrm{Z}$. If compatible within $10 \mathrm{GeV}$ with either, the event is rejected. This happens in about $2 \%$ of the cases. The events satisfying all these constraints will constitute the "signal" sample.

These requirements are not fully realistic: no flavour information will be available for light quarks, b's will be tagged only in the central part of the detector and the invariant mass of the $l \nu$ system will not be directly available. Our aim however is to define a "signal", in the same spirit as CCO3 was adopted as such at LEP 2, that is a pseudovariable which could be used to compare the results from the different collaborations. The signal is not necessarily directly observable but it should be possible to relate it via Monte Carlo to measurable quantities. If such a definition is to be useful it must correspond as closely as possible to the process which needs to be studied and the Monte Carlo corrections must be small. At this stage we want to isolate as much as possible the VV fusion signal from all other production channels, while keeping reasonably close to the experimental practice and taking into account the full set of diagrams required by gauge invariance. It becomes then possible to analyze the differences between the VV fusion signal sample and its intrinsic background. This also provides some preliminary experience at the generator level which could guide more realistic and complete studies.

In Tab. 2 the cross sections obtained for each of the described cuts are shown. The corresponding VV mass distributions are displayed in Fig. 8 and 9.

Fig. 8 shows how the invariant mass distributions for two reconstructed vector bosons is modified when the cuts described above are sequentially imposed, for the case where the Higgs boson exists and its mass is $500 \mathrm{GeV}$ and for the no-Higgs case. The no-Higgs amplitude is the amplitude in the limit $M_{H} \rightarrow \infty$. In the Unitary gauge, for the processes under investigation, this limit amounts to neglecting all diagrams with Higgs propagators. The black line refers to the full sample, the red one shows the effects of antitagging on the top. The blue line corresponds to imposing all the mentioned cuts and antitagging on the presence of three vector bosons. Fig. 9 compares the reconstructed vector boson invariant mass distribution after all cuts for the two Higgs cases ${ }^{3}$.

Since our definition of signal begins with the identification of the jets which reconstruct

\footnotetext{
${ }^{3}$ The black and red line of Fig. 9 do not reproduce the results of Fig. 6 because of a different set of cuts
} 

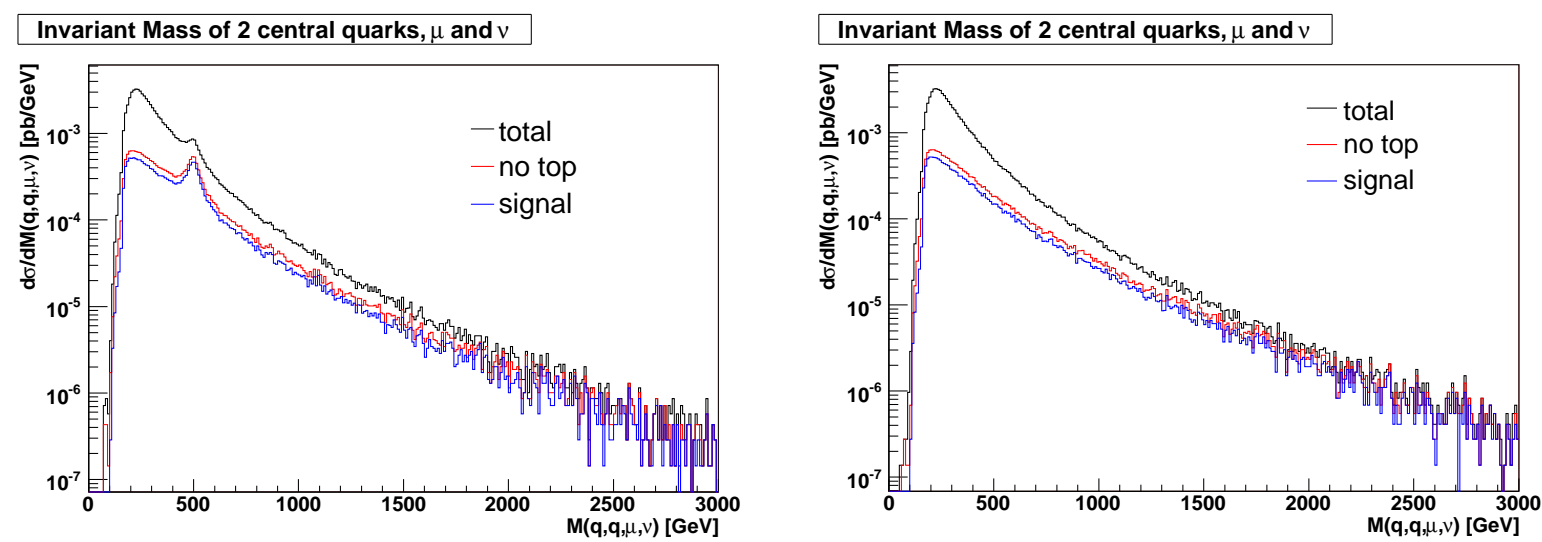

Figure 8: Distributions of the invariant mass of the two central quarks, the muon and the neutrino for $\mathrm{M}(\mathrm{H})=500 \mathrm{GeV}$ (left) and for the no-Higgs case (right). The black line refers to the full sample. The red one shows the effects of antitagging on the top. The blue line corresponds to imposing all the mentioned cuts and antitagging on the presence of three vector bosons.

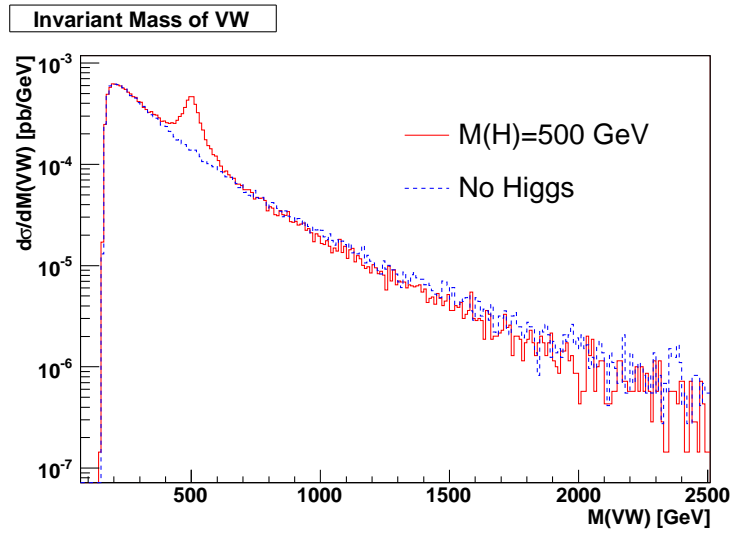

Figure 9: Distributions of the invariant mass of the two reconstructed vector bosons for $\mathrm{M}(\mathrm{H})=500 \mathrm{GeV}$ (red) and for the no-Higgs case (blue) after all cuts.In this plot the vector boson that decays hadronically is reconstructed using the two quarks that give its best mass. 

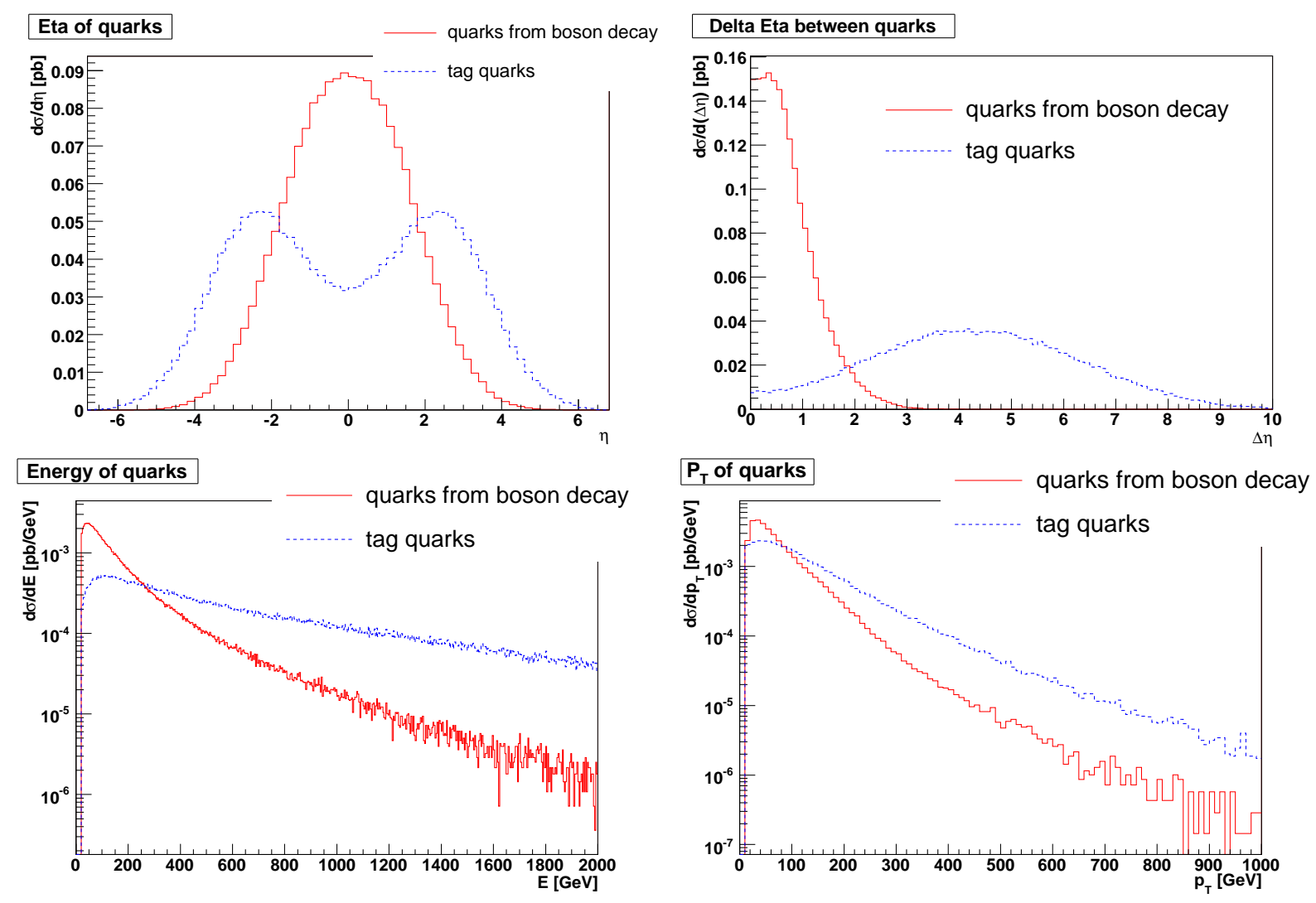

Figure 10: Distributions of the pseudorapidity $\eta$, the difference $\Delta \eta$, the energy and transverse momentum of the quarks. In red (full line) for the quarks from the decay of the vector boson and in blue (dotted line) for the tag quarks. All plots refer to $\mathrm{M}(\mathrm{H})=500$ $\mathrm{GeV}$. The distributions for the no-Higgs case lead to the same conclusions.

the EW boson rather than with the identification of the tag jets, we compare in some detail the kinematics of the quarks from a vector boson decay with the kinematics of the spectator ones. Fig. 10 shows that a good separation in phase space between the two pairs of jet is maintained. The distribution of the pseudorapidity $\eta$ of the quarks coming from the decay of the vector boson and of the tag quarks shows that the quarks from the $\mathrm{V}$ decay are, as expected, rather central (i.e. low $\eta$ ) while the spectator quarks tend to go forward/backward. Consequently the difference in pseudorapidity $\Delta \eta$ between the quarks from the bosons will be smaller than the $\Delta \eta$ between the tag quarks. The quarks from the vector boson have less energy and smaller transverse momentum with respect to the tag quarks.

It is interesting to look at possible differences in the kinematics of the VV-fusion signal with respect to the irreducible background. In Fig. 11 the "no-Higgs" case is chosen as an example, but there are no significant differences with the case of a massive Higgs. Only some variables, which are connected to the mass of the Higgs boson, show the presence of the resonance. In that case the figure for the finite mass Higgs is shown. The total invariant mass of the six fermions in the final state is presented for the signal and for the full sample: the signal tends to have a very large final six fermion mass. The muon 
from the signal has a larger $p_{T}$ than the one from the background, and the same applies to the spectator quarks. In the background the energy of the tag quarks are peaked at low values, although there is a long tail at high energy as for the signal. The transverse momentum of the $\mathrm{W}$ that decays leptonically is shown for the signal events only and for the total. We can clearly see the presence of the Higgs resonance around $p_{T} \approx M_{H} / 2$. The difference of the $\eta$ 's of the tag quarks is also shown: the signal tends to have a larger $\Delta \eta$ with respect to the background.

\begin{tabular}{|l|c|c|c|c|c|c|}
\hline \multirow{2}{*}{} & \multicolumn{2}{|c|}{ no Higgs } & \multicolumn{2}{c|}{$500 \mathrm{GeV}$} & \multicolumn{2}{c|}{$170 \mathrm{GeV}$} \\
\cline { 2 - 7 } & $\sigma(\mathrm{pb})$ & perc. & $\sigma(\mathrm{pb})$ & perc. & $\sigma(\mathrm{pb})$ & perc. \\
\hline total & 0.689 & $100 \%$ & 0.718 & $100 \%$ & 1.003 & $100 \%$ \\
\hline signal & 0.158 & $23 \%$ & 0.184 & $26 \%$ & 0.409 & $41 \%$ \\
\hline top & 0.495 & $72 \%$ & 0.494 & $69 \%$ & 0.496 & $49 \%$ \\
\hline non VV resonant & 0.020 & $3 \%$ & 0.023 & $3 \%$ & 0.040 & $4 \%$ \\
\hline three bosons & 0.016 & $2 \%$ & 0.017 & $2 \%$ & 0.057 & $6 \%$ \\
\hline
\end{tabular}

Table 2: Cross sections and percentages of events for different Higgs masses

\section{PHASE versus other Monte Carlo's}

In this section we compare PHASE with PYTHIA [17] and MADEVENT [18]. Since PHASE is the only dedicated code that uses an exact calculation for the $2 \rightarrow 6$ processes, it has been used as a reference. The PYTHIA Monte Carlo employs the Equivalent Vector Boson Approximation, and includes only longitudinally polarized vector bosons (processes 7177). The cross section in PYTHIA depends strongly on the cut applied to the $\mathrm{p}_{\mathrm{T}}$ of the outgoing W's in their center of mass. This cut, stored in $\operatorname{CKIN}(3)$, is unavoidable since it eliminates the divergence due to the photon exchange diagram in the on shell WW scattering. Notice that this divergence is an artifact of the on shell projection of the $2 \rightarrow 2$ amplitude. In the exact six fermion matrix element the photon divergence is absent. For the present comparison $\operatorname{CKIN(3)}$ was set at $50 \mathrm{GeV}$.

Fig. 12 shows the comparison between PHASE and PYTHIA for the $M_{H}=500 \mathrm{GeV}$ and for the case where no Higgs boson is produced (in PYTHIA this can be simulated by asking for a very high Higgs mass). The same signal selection cuts, including those in Tab. 1 have been applied to both PHASE and PYTHIA events. For a Higgs of $500 \mathrm{GeV}$ one finds a reasonable agreement around the resonance but a large discrepancy in the rest of the mass spectrum. For very large Higgs masses the two results are in sharp disagreement over most of the spectrum, but for the largest VV invariant masses.

As a check, in Fig. 13 the contributions to the cross section due to the different polarizations in $u d \rightarrow u d c \bar{s} \mu \bar{\nu}$ are shown for the two different scenarios. Since it is impossible to separate the polarizations of the vector bosons in the full $2 \rightarrow 6$ matrix element, we have used a dedicated code that keeps only the diagrams in which two W bosons are produced. This corresponds to convoluting the matrix element for $u d \rightarrow$ $u d W^{+} W^{-}$with the matrix elements for the polarized decay of the W's and with their Breit-Wigner distributions. This approach violates gauge invariance, but since we are 

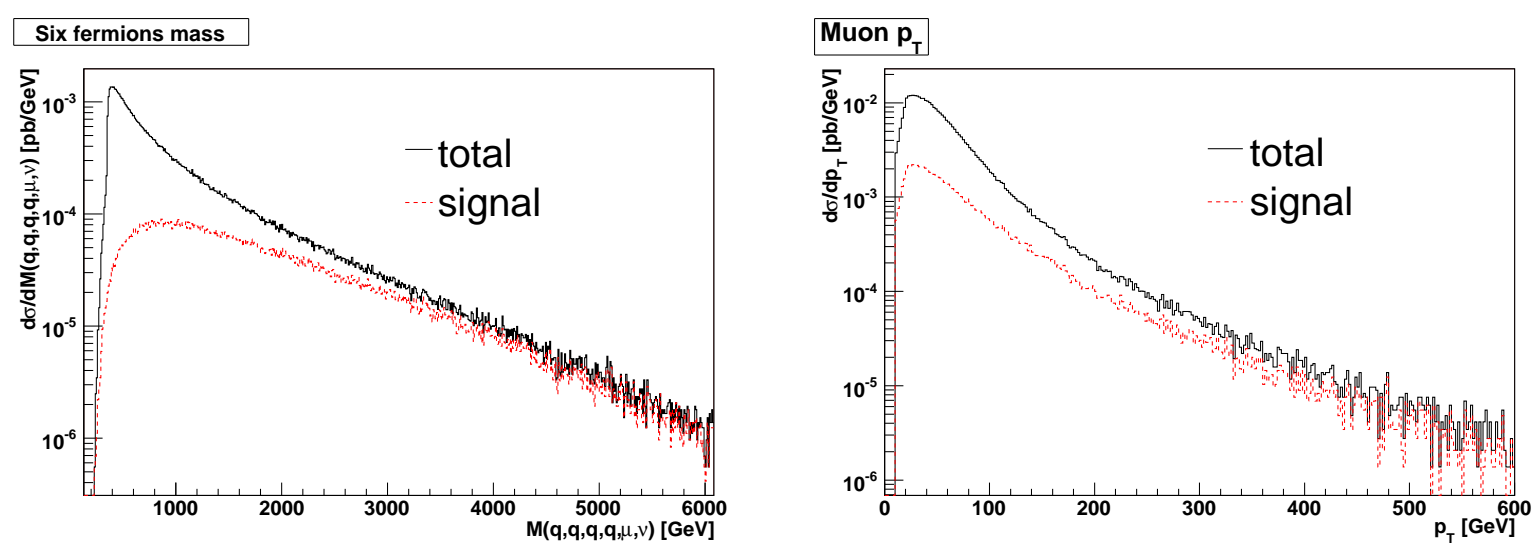

$\mathrm{P}_{\mathrm{T}}$ of tag quarks
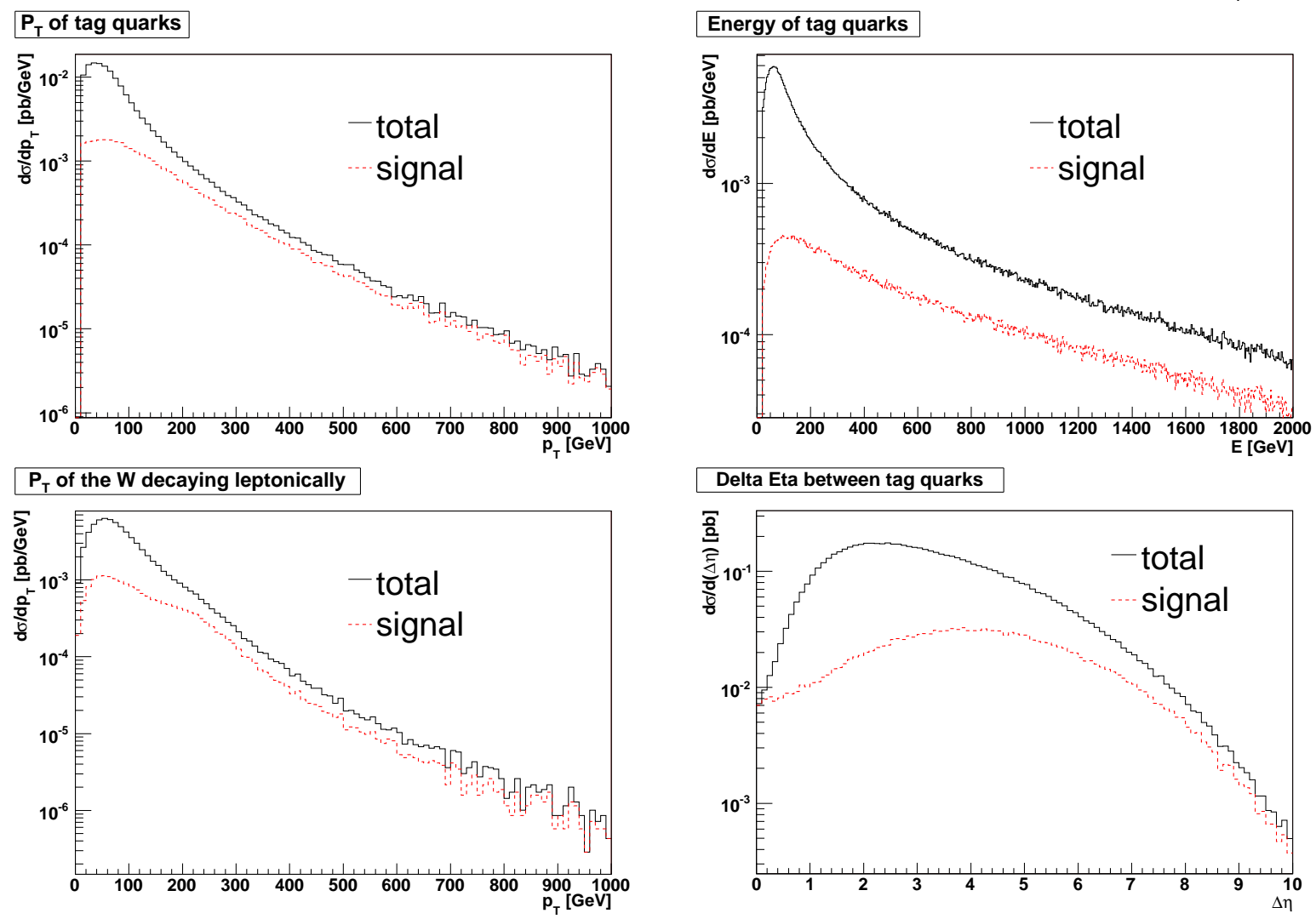

Figure 11: Differential cross section as a function of the invariant mass of the six fermions in the final state, the transverse momentum of the muon, the transverse momentum of the two tag quarks, the energy of the two forward quarks, the transverse momentum of the W decaying leptonically and the difference in pseudorapidity between the two tag quarks for all the events (black) and for the signal events (red). All plots refer to the no-Higgs case with the exception of the $p_{T}$ of the $W$ which decays leptonically which is for $\mathrm{M}(\mathrm{H})=500$ $\mathrm{GeV}$. 
restricting the W's to be close to their mass shell these effects are expected to be small. The cuts shown in Tab. 3 have been applied in this case, in order to enhance $W W \rightarrow W W$ compared to $Z Z \rightarrow W W$.

The TT cross section is essentially independent of the Higgs mass and it is the largest one, outside the peak region, for $M_{H}=500 \mathrm{GeV}$. In the no-Higgs case LL and TT production contribute equally to the spectrum. An attempt to experimentally separate LL and TT polarizations has been done and is described in Sect. 6 .

For $M_{H}=500 \mathrm{GeV}$ case the disagreement at high invariant mass is due to the fact that only LL is considered in PYTHIA, while the cross section for TT polarized vector bosons dominates. For the no-Higgs case, the cross section for LL and TT polarizations are of the same order. The agreement between PYTHIA and PHASE at high masses is probably accidental, the missing polarizations being compensated by the growth of the LL cross section in PYTHIA, which as the total center of mass energy increases gets larger contribution from the photon exchange diagram at small scattering angles. We have not pursued this issue any further since Fig. 12 makes it clear that PYTHIA is not an appropriate tool to investigate VV scattering.

\begin{tabular}{|c|}
\hline $\mathrm{E}($ quark,lepton $)>20 \mathrm{GeV}$ \\
\hline $\mathrm{p}_{\mathrm{T}}($ quark,lepton $)>10 \mathrm{GeV}$ \\
\hline $1<\eta(d)<5.5$ \\
\hline$-1>\eta(u)>-5.5$ \\
\hline $70 \mathrm{GeV}<M(c \bar{s}, \mu \bar{\nu})<90 \mathrm{GeV}$ \\
\hline
\end{tabular}

Table 3: Acceptance cuts for $u d \rightarrow u d W^{+} W^{-} \rightarrow u d c \bar{s} \mu \bar{\nu}$ in Fig. 13. The initial state $u(d)$-quark is in the $z(-z)$ direction.

MADEVENT in principle could simulate all processes using exact matrix elements. In practice it is far too slow to do so and is used in the "production times decay" approximation, i.e. computing only the processes in which two vector bosons are produced on their mass shell and then decaying them. Even if this procedure partially accounts for spin correlations, it neglects all correlations within decays. This approach overlooks a number of contributions and cannot be applied in the region below the VV threshold which will be actively investigated at the LHC in the quest for an intermediate mass range Higgs. Moreover, it leads to double counting in some regions of phase space. For instance the reaction

$$
u \bar{d} \rightarrow u \bar{d} c \bar{s} \mu^{-} \bar{\nu}
$$

would be approximated by the sum of two processes

$$
u \bar{d} \rightarrow u \bar{d} W^{+} W^{-} \rightarrow u \bar{d} c \bar{s} \mu^{-} \bar{\nu} \quad u \bar{d} \rightarrow W^{+} c \bar{s} W^{-} \rightarrow u \bar{d} c \bar{s} \mu^{-} \bar{\nu} .
$$

While this is a reasonable approximation over most of phase space since the $u \bar{d}$ pair from the first reaction will mainly be produced at large rapidity separation and the $u \bar{d}$ pair from the decay of the $W^{+}$in the second reaction will be at small separation, and as a consequence interference effects will be small, it leads to an overestimate of the cross section in the region of three vector boson production where all final state particles are rather central. 


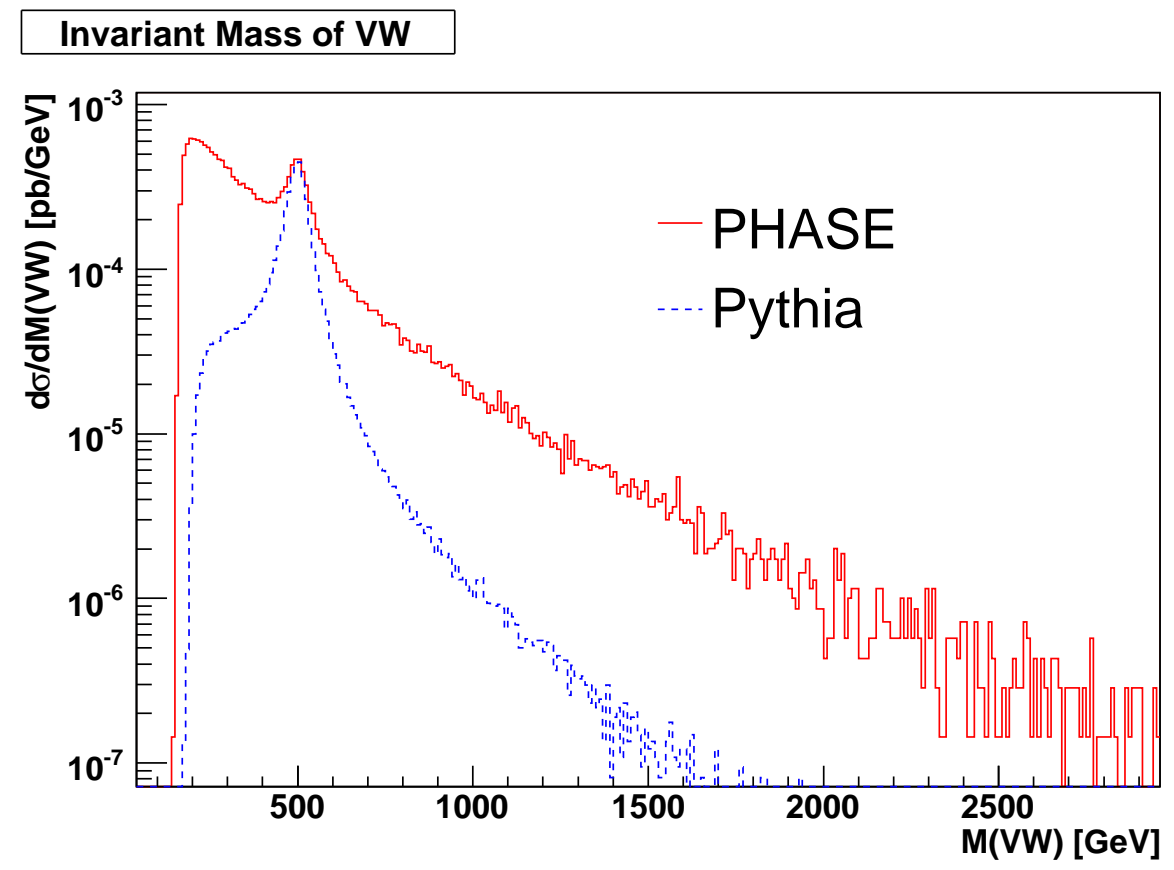

\section{Invariant Mass of VW}

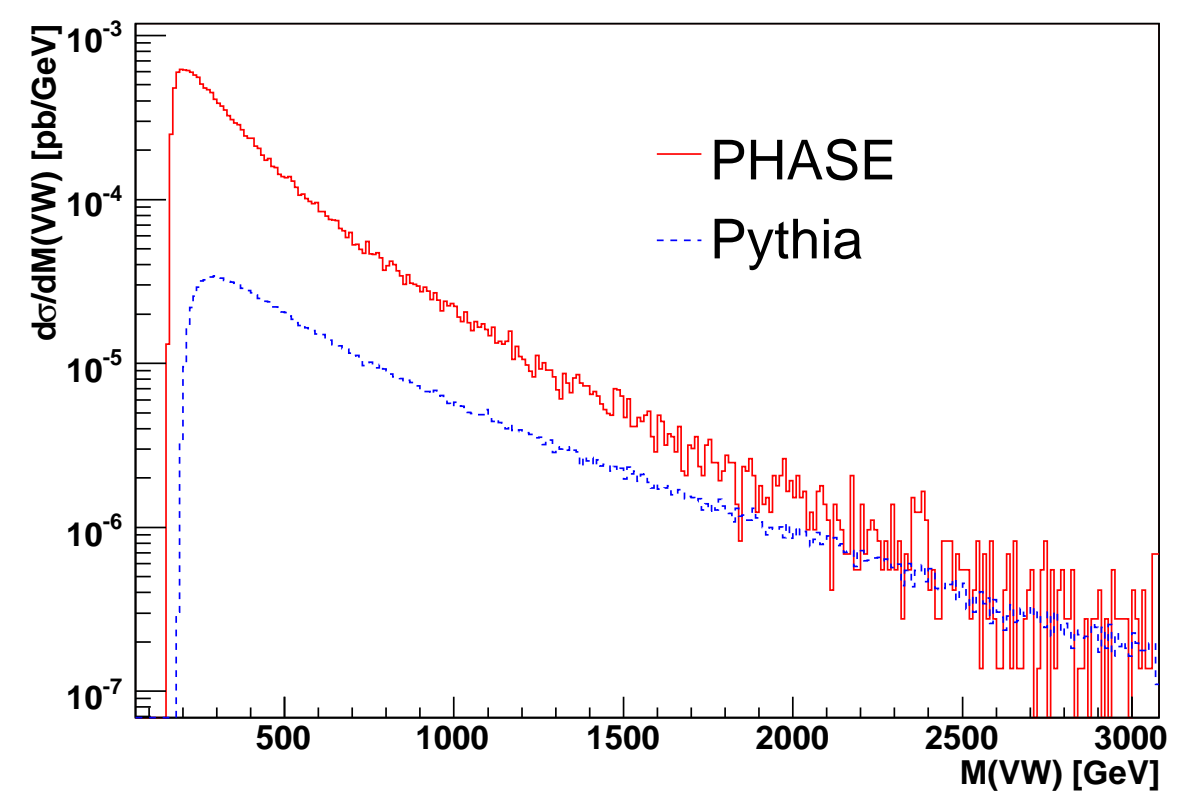

Figure 12: Cross section as a function of the invariant mass of the two vector bosons for PHASE (red) and for Pythia (blue). The upper plot refers to a Higgs mass of $500 \mathrm{GeV}$, the lower one to the no-Higgs case. 
We have compared the predictions of PHASE and those of MADEVENT for the process $u u \rightarrow u u \bar{q} q^{\prime} \mu \nu$ with $\bar{q} q^{\prime}=\bar{d} u+\bar{s} c$ and for the sum of all processes with no $b$ quarks (b's require a separate generation in MADEVENT). In this case we have adopted the scale $Q^{2}=M_{Z}^{2}$ since our standard choice Eq.(3) cannot be used in the production times decay approach. The signal has been selected as described in Sect. 3 therefore eliminating the region of three vector boson production which is not correctly described by MADEVENT. A number of distributions are shown in Fig. 14 and Fig. 15 respectively. Fig. 14 shows significant differences between the two results. The transverse momentum of the spectator quarks is harder in PHASE, as is the pseudorapidity of the reconstructed vector bosons, while the separation in pseudorapidity between the spectator quarks tends to be smaller. In PHASE the muon is more likely to fly in the direction of the $\mathrm{W}$ momentum than in MADEVENT. While none of these differences is dramatic their impact should be carefully assessed.

These differences are also present in Fig. 15, only slightly diluted in the more inclusive sample. Fig. 15 presents also the distribution of the invariant mass of the two candidate vector bosons and of the $\Delta \eta$ between the two most central quarks. At large invariant masses the two predictions coincide while close to the VV threshold MADEVENT predictions are about $7 \%$ larger. The difference in pseudorapidity between the two most central quarks is significantly harder in MADEVENT with differences of about $15 \%$ at large separation.

It should be noted that the result of the comparison between the two codes is sensitive to the mass window around the nominal mass of the EW bosons which is included. In real simulations this will depend on the necessity of rejecting backgrounds and on the uncertainty with which invariant masses of two quarks and two leptons will be reconstructed. MADEVENT predictions show no dependence on this cut, since all W's are produced exactly on mass shell. PHASE, on the contrary, takes into account the Breit-Wigner shape of the propagator squared and includes a large number of additional diagrams which become important when the vector boson are allowed to be off shell.

\section{Higgs production in PHASE}

Higgs production in VV fusion followed by Higgs decay to WW or ZZ is the second most abundant production channel over almost the full range of Higgs masses which will be explored at LHC. It is regarded as the channel with the highest statistical significance for an intermediate mass Higgs [19]. PHASE is capable of simulating Higgs production in VV fusion together with all its EW irreducible background for any Higgs mass and is particularly useful in the intermediate mass range where only one of the vector bosons can be approximately treated in a production times decay approach. For an intermediate mass Higgs the dilepton final state $H \rightarrow W W^{(*)} \rightarrow l \nu l \nu$ is slightly favored with respect to the $H \rightarrow W W^{(*)} \rightarrow l \nu j j$ channel because of the $W+n j$ background which affects the latter. In both cases the main background comes from $t \bar{t}$ production followed in importance by EW $W W j j$ production which is estimated to be about $10 \%$, much larger than QCD $W W j j$ production. The latter can be reduced with a central jet veto which does not affect the former.. The cross section after selection cuts is of the order of $1 \div 4 \mathrm{fb}$ and with such a small number of events it is important to have a precise simulation of the complete final state with full spin correlations which are complicated by the fact that in this range of Higgs masses one of the $W$ is far off shell. 
In Fig. 16 we present a number of kinematical distributions for $\mathrm{M}(\mathrm{H})=140 \mathrm{GeV}$ with different selection procedures. The green line refers to identifying the two most central quarks in pseudorapidity as the quarks from $\mathrm{W}$ decay while the black histogram refers to selecting the two quarks among the four that have the mass closest to the $\mathrm{W}$ boson mass. The blue line is obtained selecting the quark pairs which have the correct flavour content to be produced in a $\mathrm{W}$ decay and which have the mass closest to the $\mathrm{W}$ mass. The first procedure, which is also the most robust from an experimental point of view, agrees well with the third one, which is more appropriate from a theoretical perspective but which can only be applied at generator level, over the full range of all variables. It should be pointed out that the actual ratio of signal over background depends cruciallly on the experimental resolution and on the set of additional selection cuts [19]. For reference, the invariant mass distribution in Fig. 16 is plotted with a bin size of $10 \mathrm{GeV}$.

\section{The high mass region}

An interesting physics possibility is to investigate whether there exist or not an elementary Higgs boson by measuring the VW cross section at large M(VW). Preliminary studies performed at CMS with a fast detector simulation [20] showed that a resolution of about $10-15 \%$ on $\mathrm{M}(\mathrm{VV})$ up to $2 \mathrm{TeV}$ is achievable with about $100 \mathrm{fb}^{-1}$. The rise of the cross section related to unitarity violation in the no-Higgs case is difficult to detect at the LHC, since the center-of-mass energy is still rather low and the decrease of the proton distribution functions at large $x$ has the dominating effect. As we discussed in the previous section, if $\mathrm{W}_{\mathrm{L}} \mathrm{W}_{\mathrm{L}}$ final states could be isolated, the difference in the cross section at high WW masses would be sizeable, since the $W_{L} W_{L}$ cross section decreases much more rapidly in the presence of a Higgs particle than in the no-Higgs situation. In order to distinguish $\mathrm{W}_{\mathrm{L}} \mathrm{W}_{\mathrm{L}}$ from $\mathrm{W}_{\mathrm{T}} \mathrm{W}_{\mathrm{T}}$ we must exploit the different behaviour of the final state in the two cases.

To this purpose kinematical distributions for $\mathrm{M}(\mathrm{H})=170 \mathrm{GeV}, \mathrm{M}(\mathrm{H})=500 \mathrm{GeV}$ and the no-Higgs case have been compared for $\mathrm{M}(\mathrm{VV})>800 \mathrm{GeV}$ since the cross section at large $\mathrm{M}(\mathrm{VV})$ for $\mathrm{M}(\mathrm{H})=170 \mathrm{GeV}$ and $\mathrm{M}(\mathrm{H})=500 \mathrm{GeV}$ is essentially due to transversely polarized vector bosons, while the cross section for the no-Higgs case is due to a mixture of the two polarizations as shown in Fig. 13. Fig. 17 shows that the distributions are quite insensitive to the value of the Higgs mass provided it is much smaller than the invariant mass of the VV system. This raises the interesting possibility of defining Standard Model predictions for high invariant mass production of VV pairs. These predictions will obviously suffer from the usual PDF and scale uncertainties, which could however in principle be controlled by comparing with the cross section of some appropriate "standard candle" process.

We have tried several sets of cuts and we believe that using Neural Network is the most effective way of increasing the separation between the no-Higgs case and the presence of a relatively light Higgs. Two samples of events with a high invariant mass VW pair, for $\mathrm{M}(\mathrm{H})=500 \mathrm{GeV}$ and the no-Higgs case respectively, have been employed to train a Neural Network. All events satisfy the cuts in Tab. 1. A set of variables which discriminate between the two Higgs hypotheses have been used in the training. We have chosen four weakly correlated variables: the difference in pseudorapidity between the two bosons and between the two tag quarks, the transverse momentum of the tag quarks and the cosine 
of the angle between the lepton and the $\mathrm{W}$ boson in the $\mathrm{W}$ center of mass system. These (and other) kinematical variables are shown in Fig. 17 for the no-Higgs case and for $\mathrm{M}(\mathrm{H})=500 \mathrm{GeV}$ and $\mathrm{M}(\mathrm{H})=170 \mathrm{GeV}$.

\begin{tabular}{|l|c|c|c|c|c|}
\cline { 2 - 5 } \multicolumn{1}{c|}{} & \multicolumn{2}{c|}{ no-Higgs case } & \multicolumn{2}{c|}{ m(Higgs) $=500 \mathrm{GeV}$} & \multicolumn{1}{c}{} \\
\cline { 2 - 5 } \multicolumn{1}{c|}{} & $\sigma$ & $\mathcal{L}=100 \mathrm{fb}^{-1}$ & $\sigma$ & $\mathcal{L}=100 \mathrm{fb}^{-1}$ & ratio \\
\hline all events & $13.6 \mathrm{fb}$ & $1360 \pm 37$ & $11.6 \mathrm{fb}$ & $1160 \pm 34$ & 1.2 \\
\hline $\mathrm{NN}>0.52$ & $3.93 \mathrm{fb}$ & $393 \pm 20$ & $2.70 \mathrm{fb}$ & $270 \pm 16$ & 1.5 \\
\hline $\mathrm{NN}>0.54$ & $3.17 \mathrm{fb}$ & $317 \pm 18$ & $1.95 \mathrm{fb}$ & $195 \pm 14$ & 1.6 \\
\hline $\mathrm{NN}>0.56$ & $2.67 \mathrm{fb}$ & $267 \pm 16$ & $1.47 \mathrm{fb}$ & $147 \pm 12$ & 1.8 \\
\hline $\mathrm{NN}>0.58$ & $2.28 \mathrm{fb}$ & $228 \pm 15$ & $1.13 \mathrm{fb}$ & $113 \pm 11$ & 2.0 \\
\hline
\end{tabular}

Table 4: Integrated cross section for $\mathrm{M}(\mathrm{VW})>800 \mathrm{GeV}$ and number of expected events after one year at high luminosity.

The neural network, a multilayer perceptron with BFGS training, available in the ROOT package [21], takes these four variables as input, has two intermediate layers, with eight and four neurons respectively, and one output variable. The differential cross section $d \sigma / d N N$ where NN is the neural network output variable is shown on the left hand side of Fig. 18 for two event samples corresponding to the different Higgs masses. Applying a cut on NN we can enhance the separation between the heavy and light Higgs case. The corresponding purity and efficiency is shown on the right hand side.

In Tab. 4 the integrated cross section and the number of events for the two Higgs cases for $\mathrm{M}(\mathrm{VW})>800 \mathrm{GeV}$ are shown for different values of the cut. The corresponding ratio is also presented. The predictions for $\mathrm{M}(\mathrm{H})=500 \mathrm{GeV}$ have a statistical uncertainty of about $10 \%$ for $\mathrm{NN}>0.56$, while the very heavy Higgs case, with all the necessary caveats, predicts a cross section which is larger by about a factor of two. This is quite encouraging and suggests that further study should be worthwhile in order to sharpen the SM predictions.

In Fig. 19 we show the ratio

$$
\frac{\int_{M_{c u t}}^{\infty} d M_{V W} \frac{d \sigma_{\text {noHiggs }}}{d M_{V W}}}{\int_{M_{c u t}}^{\infty} d M_{V W} \frac{d \sigma_{M_{H}=500}}{d M_{V W}}}
$$

for different cuts on the neural network output variable as a function of $M_{\text {cut }}$, the lower limit of the integration range on $M_{V W}$. The difference between the two cross sections is concentrated at large values of NN and increases with increasing values of the output variable. With a fixed lower cut on $\mathrm{NN}$ the ratio between integrated cross sections increases with $M_{\text {cut }}$. With $M_{\text {cut }}>1 \mathrm{TeV}$ and NN $>0.56$ the cross section is about $2 \mathrm{fb}$ for a very heavy Higgs, roughly twice the expected yield for a light Higgs scalar. On the right hand side of Fig. 19 we show the ratio 6 for different group of processes. The set which includes $W^{ \pm} W^{ \pm}$is the one with the largest separation, while the sets including $Z Z$ and $Z W$ scattering are less sensitive to the cut on $\mathrm{NN}$. 


\section{Summary and future}

In this paper we have studied all $q_{1} q_{2} \rightarrow q_{3} q_{4} q_{5} q_{6} l \nu$ processes at order $\mathcal{O}\left(\alpha_{e m}^{6}\right)$ at the LHC using for the first time a full fledged six fermion Monte Carlo event generator. We have examined how simple kinematical cuts can be applied at generator level to extract the VV signal from the irreducible background. In the high mass region we have compared the case of a relatively light Higgs with the no-Higgs case as a guide to separate the LL component of VV scattering. Employing a neural network approach it seems possible to obtain a good separation of the two cases.

Work is in progress to extend PHASE to cover qqZZ final states. The calculation of the full set of processes $2 \rightarrow 6$ at $\mathcal{O}\left(\alpha_{s}^{2} \alpha_{e m}^{4}\right)$ is under way. A phenomenological study of $\mathrm{VV}$ processes with both vectors decaying leptonically is in project.

\section{References}

[1] Proceedings of the Large Hadron Collider Workshop, Aachen 1990, CERN Report 90-10, G. Jarlskog and D. Rein (eds.).

[2] A. Djouadi, The Anatomy of Electro-Weak Symmetry Breaking. Tome I: The Higgs in the Standard Model, [hep-ph/0503172].

[3] ATLAS Collaboration, Detector and Physics Performance Technical Design Report, Vols. 1 and 2, CERN-LHCC-99-14 and CERN-LHCC-99-15.

[4] K.A. Assamagan, M. Narain, A. Nikitenko, M. Spira, D. Zeppenfeld (conv.) et al., Report of the Higgs Working Group, Proceedings of the Les Houches Workshop on "Physics at TeV Colliders", 2003, [hep-ph/0406152].

[5] The LEP Collaborations (ALEPH, DELPHI, L3 and OPAL), the LEP Electroweak Working Group and the SLD Heavy Flavour Group, A combination of preliminary Electroweak measurements and constraints on the Standard Model, [hepex/0412015]; http://lepewwg.web.cern.ch/LEPEWWG.

[6] M.E. Peskin, J.D. Wells, Phys. Rev. D64 (2001) 093003, [hep-ph/0101342].

[7] M.S. Chanowitz, Strong WW scattering at the end of the 90's: theory and experimental prospects. In Zuoz 1998, Hidden symmetries and Higgs phenomena 81-109. [hep-ph/9812215]

[8] J. Bagger et al., Phys. Rev. D52 (1995) 3878; A. Dobado, M.J. Herrero, J.R. Peláez and E. Ruiz Morales, Phys. Rev. D62 (2000) 055011, [hep-ph/9912224]; J.M. Butterworth,B.E. Cox and J.R. Forshaw, Phys. Rev. D65 (2002) 96014. [hep-ph/0201098]

[9] M.J. Duncan, G.L. Kane and W.W. Repko, Nucl. Phys. B272 (1986) 517; D.A. Dicus and R. Vega, Phys. Rev. Lett. 57 (1986) 1110; J.F. Gunion, J. Kalinowski and A. Tofighi-Niaki, Phys. Rev. Lett. 57 (1986) 2351.

[10] R.N. Cahn, S.D. Ellis, R. Kleiss and W.J. Stirling, Phys. Rev. D35 (1987) 1626; V. Barger, T. Han and R. Phillips, Phys. Rev. D37 (1988) 2005 and D36 (1987) 
295; R. Kleiss and J. Stirling, Phys. Lett. 200B (1988) 193; V. Barger et al., Phys. Rev. D42 (1990) 3052; ibid. Phys. Rev. D44 (1991) 1426; ibid. Phys. Rev. D46 (1992) 2028; D. Froideveaux, in Ref. [1] Vol II, p. 444; M. H. Seymour, ibid., p. 557; U. Baur and E.W.N. Glover, Phys. Lett. B252 (1990) 683; D. Dicus, J. Gunion and R. Vega, Phys. Lett. B258 (1991) 475; D. Dicus, J. Gunion, L. Orr and R. Vega, Nucl. Phys. B377 (1991) 31; J. Bagger et al.,Phys. Rev. D49 (1994) 1246;V. Barger, R. Phillips and D. Zeppenfeld, Phys. Lett. B346 (1995) 106; J. Bagger et al.,Phys. Rev. D52 (1995) 3878;K. Iordanidis and D. Zeppenfeld, Phys. Rev. D57 (1998) 3072; R. Rainwater and D. Zeppenfeld, Phys. Rev. D60 (1999) 113004; erratum ibid D61 (2000) 099901.

[11] M.S. Chanowitz and M.K. Gaillard, Nucl. Phys. B261 (1985) 379. M.S. Chanowitz and M.K. Gaillard, Phys. Lett. 142B, 85 (1984) and ref. 1; G. Kane, W. Repko, B. Rolnick, Phys. Lett. B148, 367 (1984); S. Dawson, Nucl. Phys. B29 (1985) 42.

[12] E. Accomando, A. Ballestrero, E. Maina, JHEP 0507 (2005) 016, [hep-ph/0504009].

[13] A. Ballestrero and E. Maina, Phys. Lett. B350 (1995) 225, [hep-ph/9403244].

[14] A. Ballestrero, PHACT 1.0 - Program for Helicity Amplitudes Calculations with Tau matrices' [hep-ph/9911318] in Proceedings of the 14th International Workshop on High Energy Physics and Quantum Field Theory (QFTHEP 99), B.B. Levchenko and V.I. Savrin eds. (SINP MSU Moscow), pg. 303.

[15] E. Accomando, A. Ballestrero, E. Maina, Talk given at 9th International Workshop on Advanced Computing and Analysis Techniques in Physics Research (ACAT 03), Tsukuba, Japan, 1-5 Dec 2003. Nucl.Instrum.Meth.A534:265-268,2004, [hepph/0404236]; E. Accomando, A. Ballestrero, E. Maina, Proceedings of 18th International Workshop on High-Energy Physics and Quantum Field Theory (QFTHEP 2004), St. Petersburg, Russia, 17-23 Jun 2004. [hep-ph/0505225]

[16] CTEQ Coll.(H.L. Lai et al.) Eur. Phys. J. C12 (2000) 375.

[17] T. Sjöstrand et al., Comp. Phys. Commun. 135 (2001) 238, [hep-ph/0010017];

T. Sjöstrand, L. Lönnblab and S. Mrenna, [hep-ph/0108264].

[18] F. Maltoni, T. Stelzer, JHEP 0302 (2003) 027; T. Stelzer and W. F. Long, Comput. Phys. Commun. 81 (1994) 357;

H. Murayama, I. Watanabe and K. Hagiwara, KEK-91-11.

[19] S. Asai et al., Eur.Phys.J.C32S2:19-54,2004, [hep-ph/0402254].

[20] R. Bellan, PhD Thesis, http://www.to.infn.it/ bellan/works/tesi/tesi_bellan.pdf

[21] http://root.cern.ch 

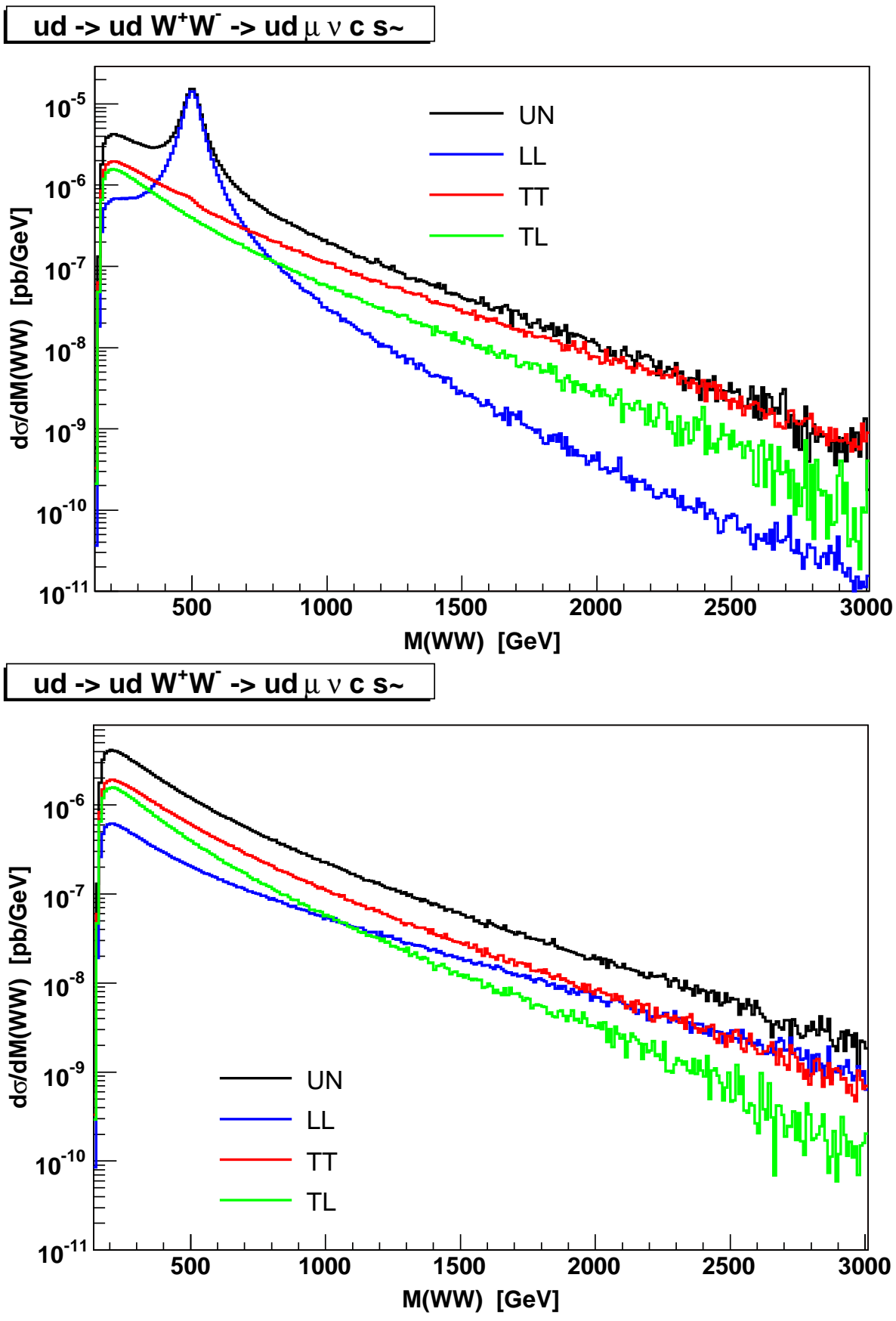

Figure 13: Cross section as a function of the invariant mass of the two vector bosons for their different polarizations. The upper plot refers to a Higgs mass of $500 \mathrm{GeV}$, the lower one to the no-Higgs case. 

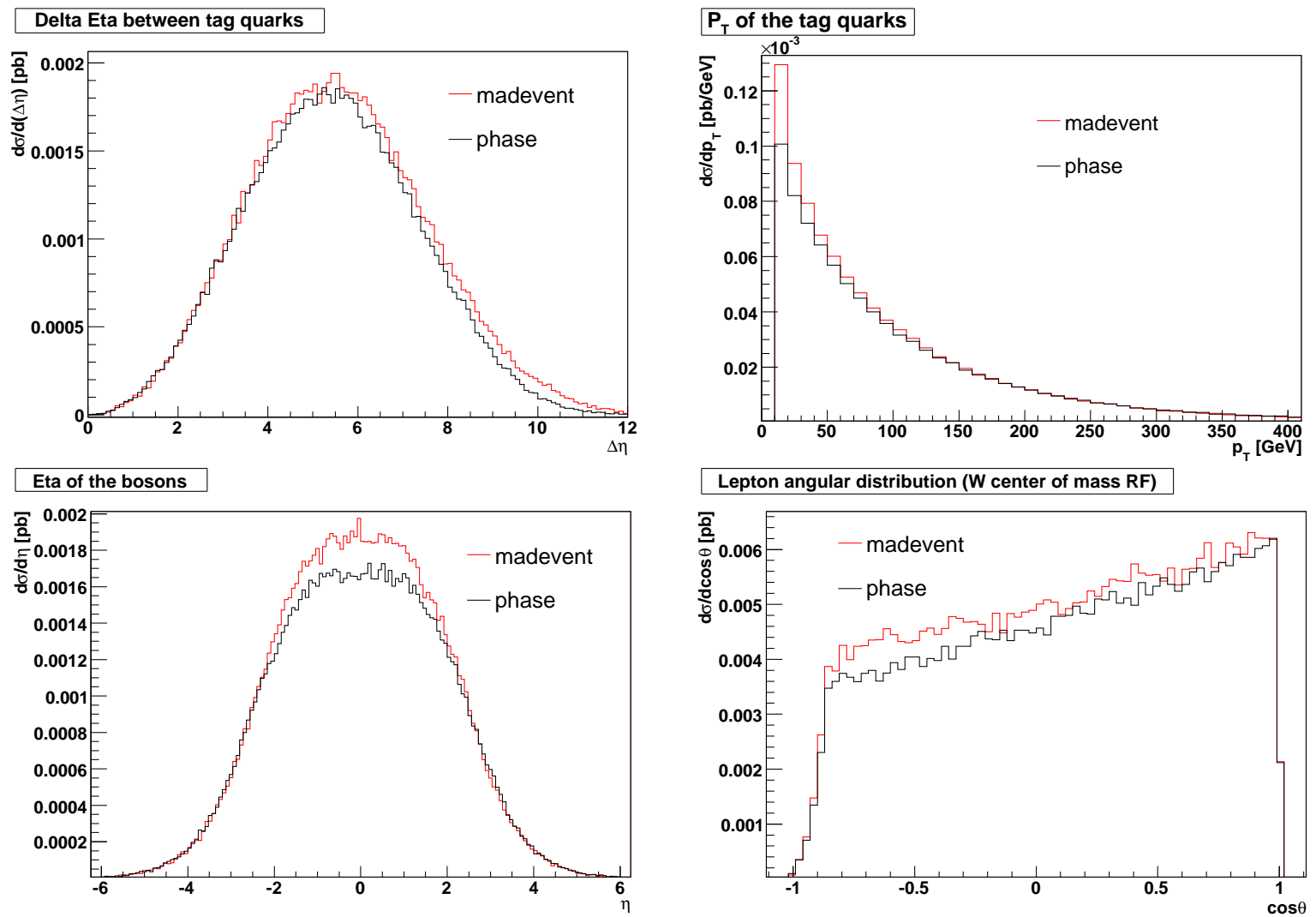

Figure 14: Cross section for the processes $u u \rightarrow u u \bar{q} q^{\prime} \mu \nu$ with $\bar{q} q^{\prime}=\bar{d} u+\bar{s} c$ as a function of the separation in pseudorapidity between the spectator quarks, their transverse momentum, the pseudorapidity of the reconstructed vector bosons and the angle of the muon with respect to the $\mathrm{W}$ direction of flight in the $\mathrm{W}$ rest frame for PHASE (black) and for MADEVENT (red) for a Higgs mass of $500 \mathrm{GeV}$. 

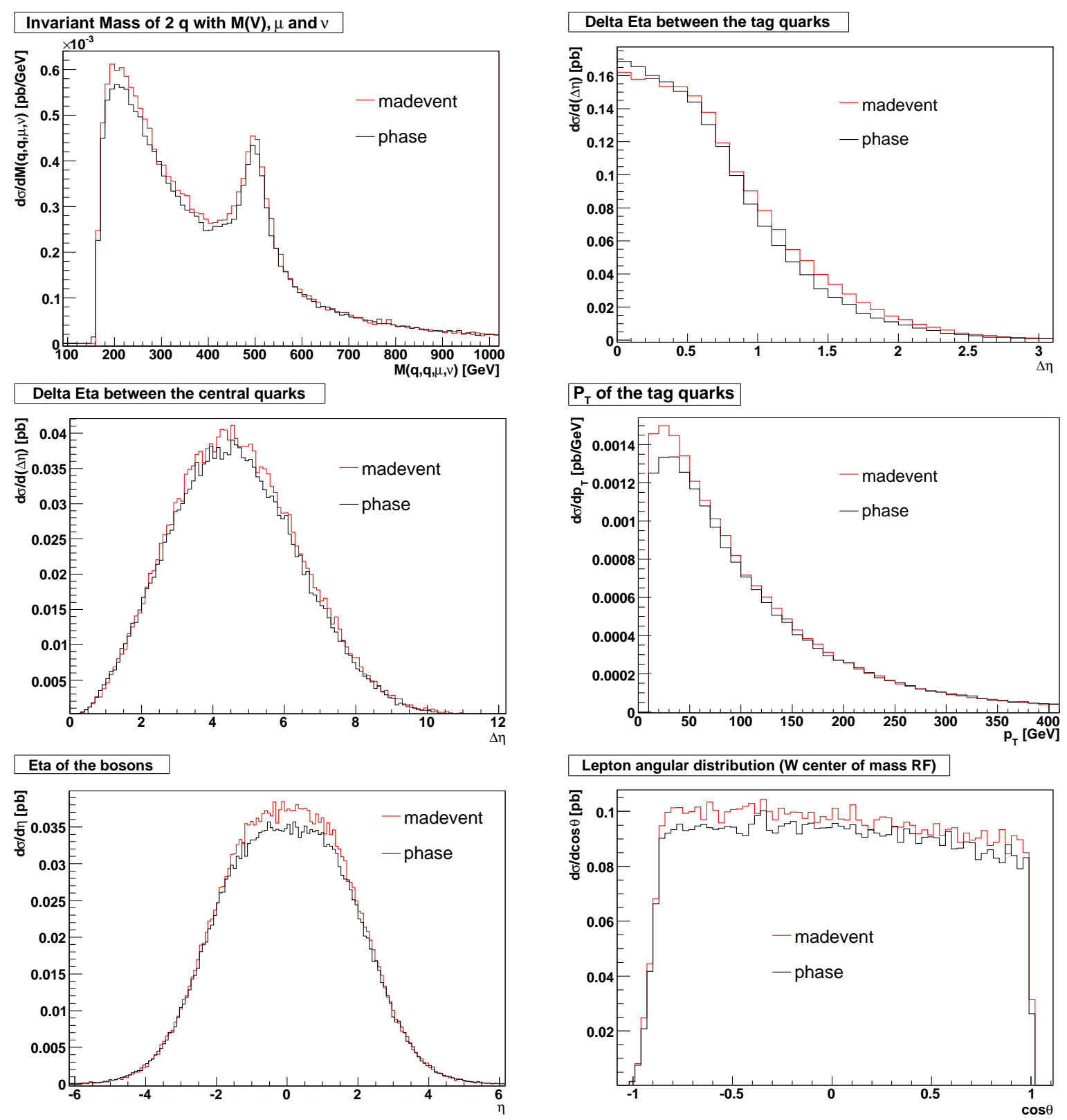

Figure 15: Cross section for the full sample as a function of the invariant mass of the two candidate vector bosons, the $\Delta \eta$ between the two most central quarks, the $\Delta \eta$ between spectator quarks, their transverse momentum, the pseudorapidity of the reconstructed vector bosons and the angle of the muon with respect to the $\mathrm{W}$ direction of flight in the W rest frame for PHASE (black) and for MADEVENT (red) for a Higgs mass of $500 \mathrm{GeV}$. 

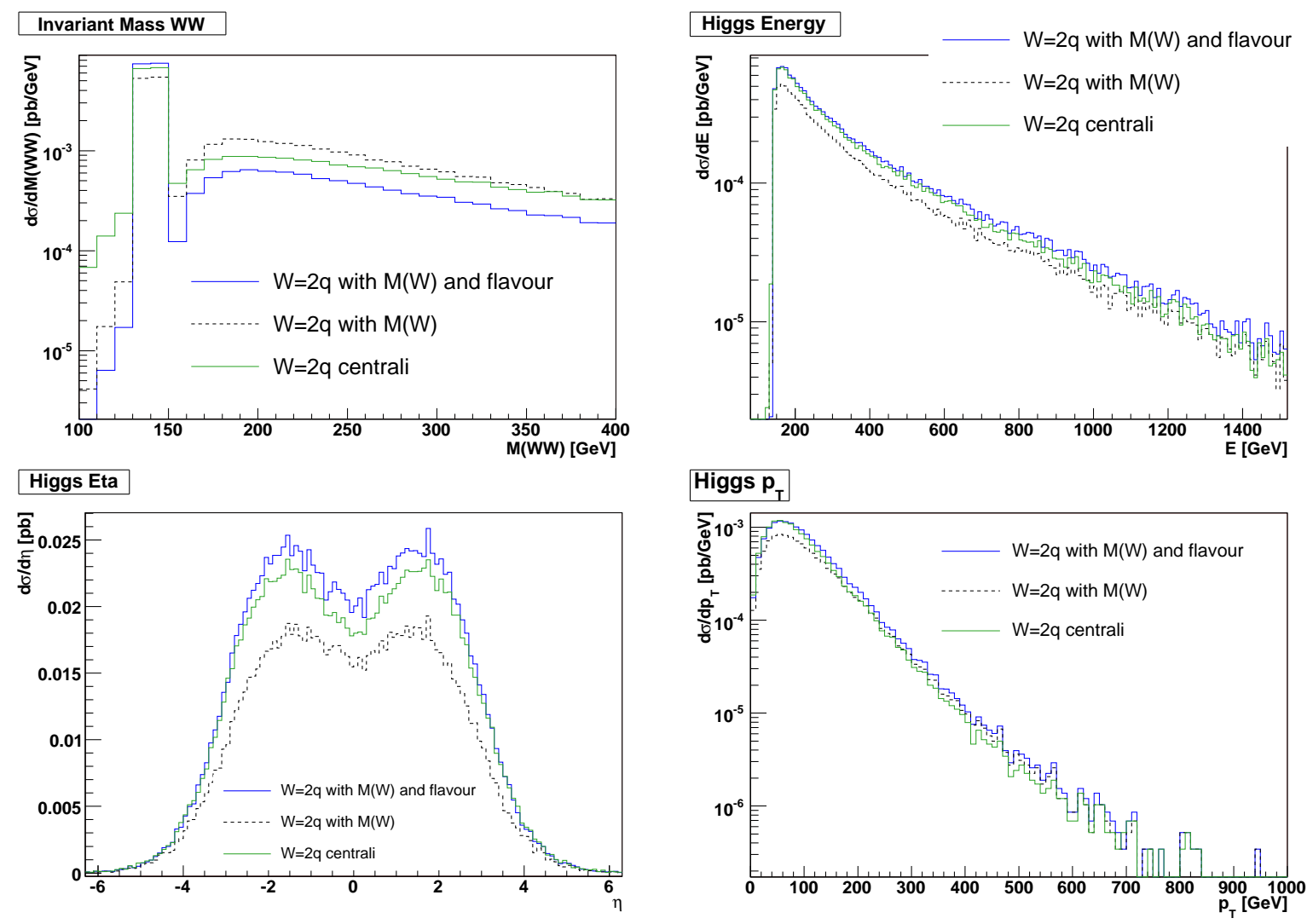

Figure 16: Distribution of the invariant mass $M_{\mathrm{VV}}$ of the two candidate vector bosons, their energy, pseudorapidity and transverse momentum for a Higgs mass of $140 \mathrm{GeV}$. In the last three plots only events with $120<M_{\mathrm{VV}}<160 \mathrm{GeV}$ are included. 

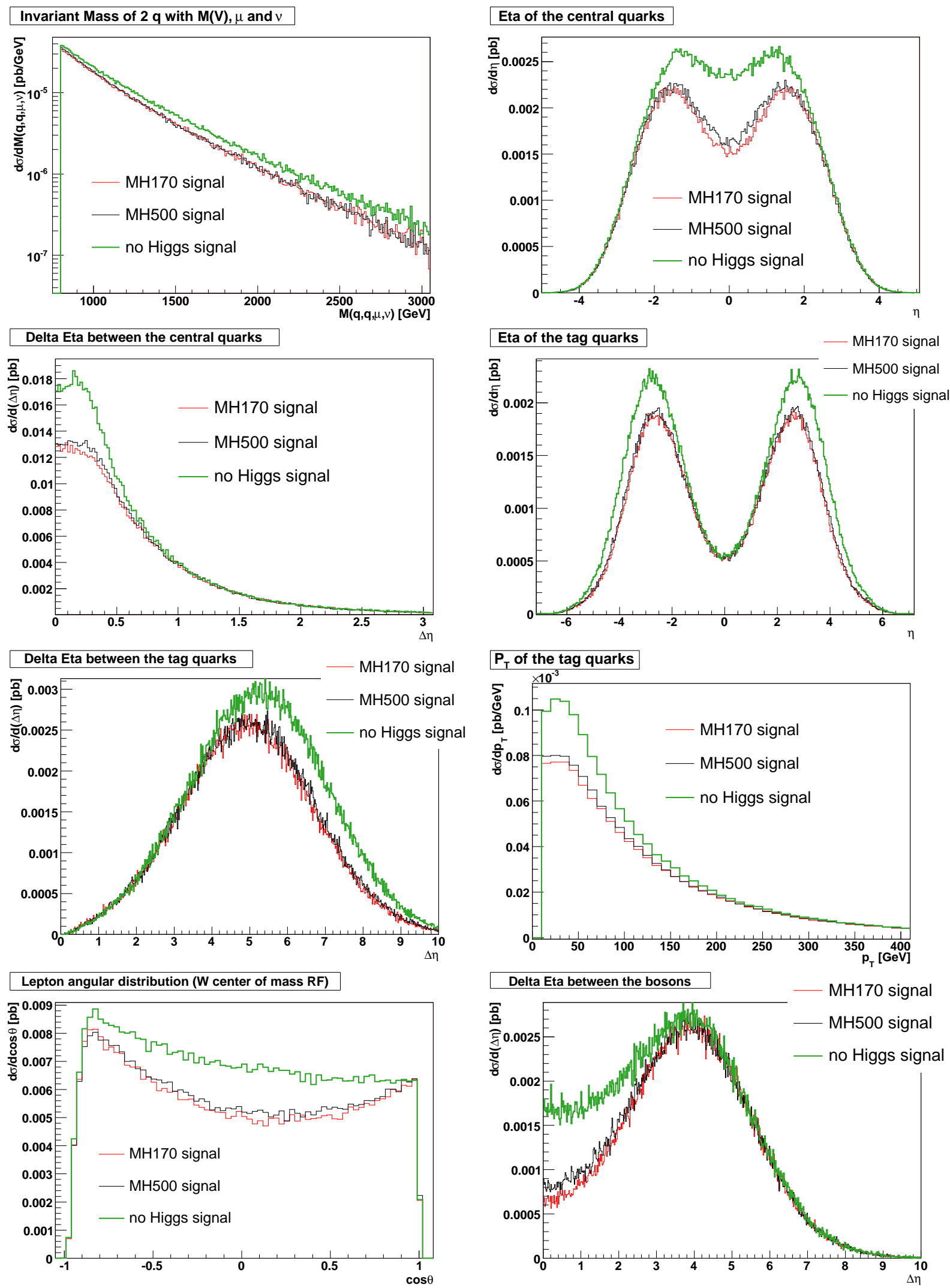

Figure 17: The invariant mass of the two vector bosons, the pseudorapidity $\eta$ of the two central jets, the $\Delta \eta$ of the two central jets, the $\eta$ of the forward quarks the $\Delta \eta$ of the two forward quarks, the transverse momentum of the forward quarks, the $\Delta \eta$ of the two vector bosons, the cosine of the angle between the lepton and the $\mathrm{W}$ boson in the $\mathrm{W}$ boson rest frame for $M(V W)>800 \mathrm{GeV}$. In green (full line) for the no-Higgs case, in black (dashed) for $\mathrm{M}(\mathrm{H})=500 \mathrm{GeV}$ and in red for $\mathrm{M}(\mathrm{H})=170 \mathrm{GeV}$. The last four variables are used as input to the neural net. 

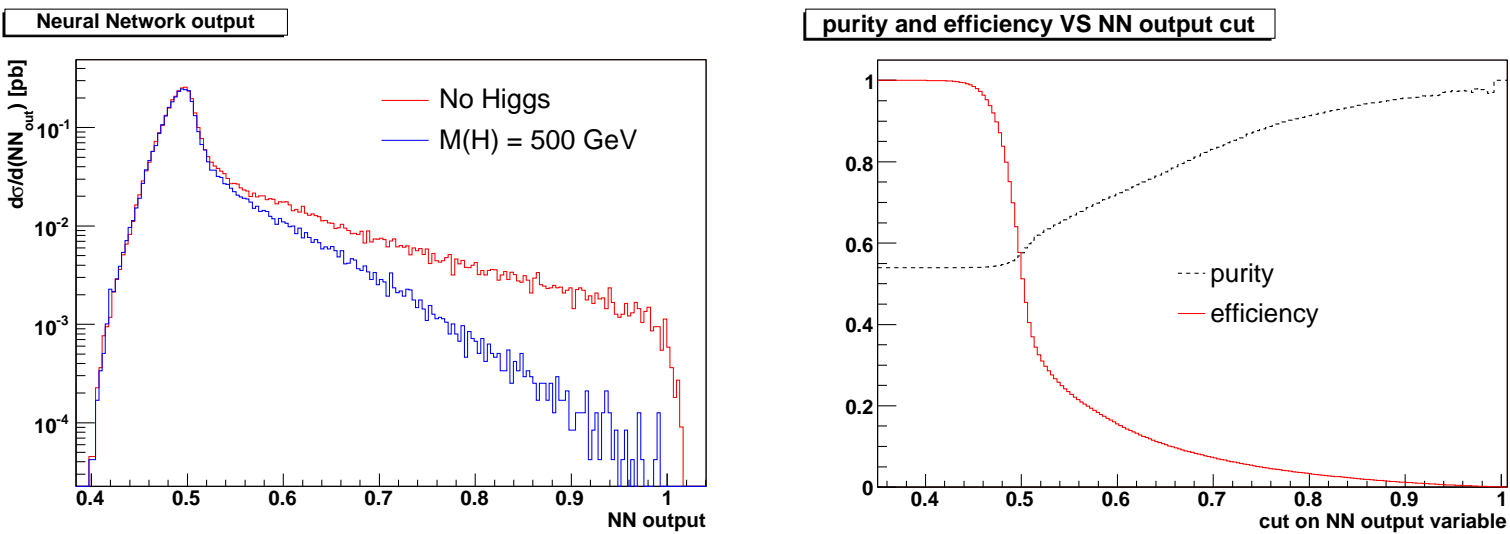

Figure 18: $d \sigma / d N N$ where $N N$ is the neural network output variable. in red (full line) for the no-Higgs case and in black (dashed) for $\mathrm{M}(\mathrm{H})=500 \mathrm{GeV}$ on the LHS. The corresponding purity and efficiency on the RHS
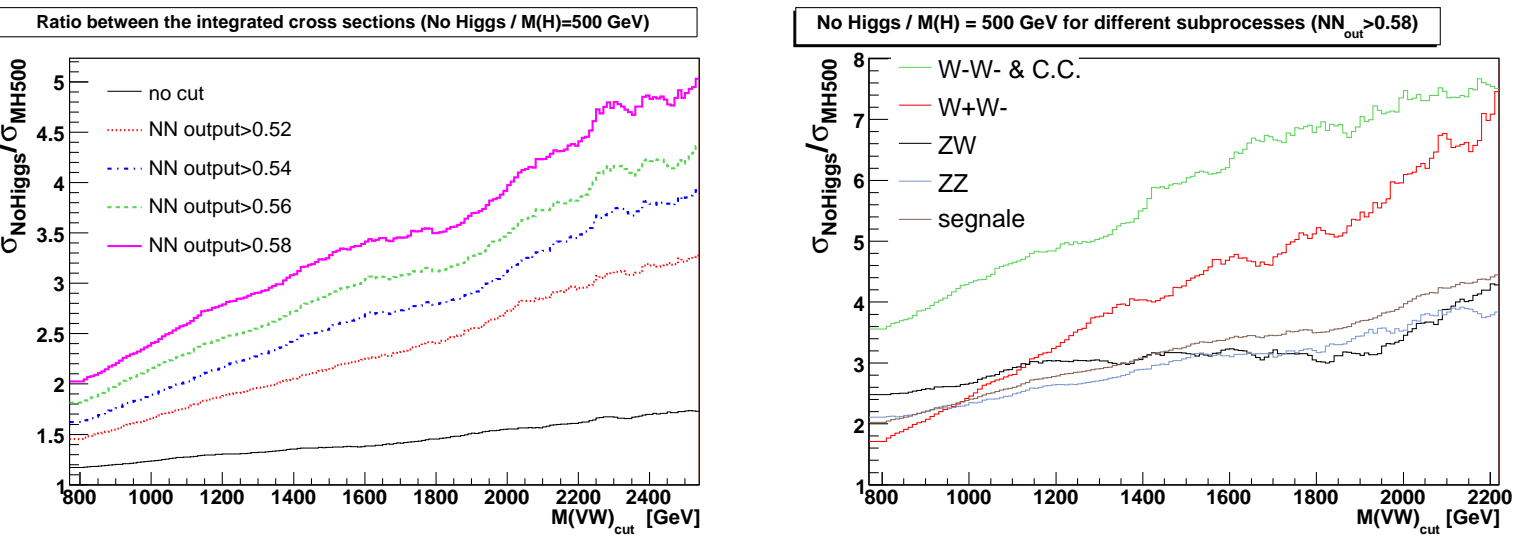

Figure 19: On the left, the ratio of cross sections, integrated between $M_{c u t}$ and infinity, as a function of $M_{\text {cut }}$, for different values of the neural network variable. On the right,the ratio Eq.(6) for different groups of processes under the condition $\mathrm{NN}>0.58$ 